\title{
CHRocodile CLS : capteur chromatique ligne dédié à la métrologie haute cadence et haute résolution
}

\author{
D. Dupraz ${ }^{1, a}$ \\ ${ }^{1}$ PRECITEC, Chemin de la Muscatelle 13790 Châteauneuf-le-Rouge, France
}

\begin{abstract}
The high resolution and high-speed 3D measurement is of great interest for a wide range of applications. The CHRocodile line sensor is a multi-point optical sensor dedicated to non-contact surface measurement. This sensor is based on confocal chromatic principle which authorizes high resolution and high speed thickness and altitude measurement. The CHRocodile CLS has an original architecture with no exposed optical cable. PRECITEC is the first company to propose a confocal chromatic device, with all elements embedded in one optoelectronic unit. With this unique architecture, CHRocodile CLS sensor is ideally suited to industrial inline 3D inspection and industrial changing requirement.
\end{abstract}

\section{Capteurs et métrologie 3D}

Dans le domaine de la mesure 3D, le choix du capteur embarqué dans une machine de mesure, une machine spéciale ou directement au-dessus d'un convoyeur est primordial pour assurer une mesure optimale de distance, de rugosité ou de topographie. Selon la nature de l'objet à mesurer (dimension, état de surface, forme, matériaux utilisés), le type de contrôle (100\% ou échantillonnage, laboratoire ou production) et le niveau de performances attendues (résolution, cadence, distance de travail), certaines méthodes doivent être privilégiées par rapport à d'autre. Les capteurs à contact par exemple inexploitables pour des mesures sur des matériaux déformables ou lors de mesure en défilement. Les capteurs optiques sont des solutions par nature sans contact qui trouvent un intérêt grandissant dans le domaine du contrôle industriel. Ainsi les distances de travail élevées et la capacité à mesurer sur tout type de matériaux offre à l'utilisateur une flexibilité essentielle.

\section{Capteurs optiques}

Les capteurs optiques sont souvent proposés comme des options sur des machines de mesure 3D ou des MMT (machine à mesurer tridimensionnelle) afin de rendre les systèmes les plus polyvalentes possibles (offre multicapteurs). Les méthodes optiques utilisées pour la mesure tridimensionnelle sont la triangulation laser, l'imagerie confocale chromatique, l'interférométrie, la projection de franges, la variation de focus etc... Chacune d'elles possède un champ d'application qui lui est généralement dédié même si quelques recouvrements sont possibles. En complément de la vision industrielle, le laser est une solution souvent exploitée pour l'inspection car le coût d'un tel capteur est relativement faible. En revanche, les phénomènes d'ombrage (triangulation), la résolution latérale faible (taille de spot) ou les effets speckle (granulosité sur métal par exemple) nécessitent d'utiliser dans certains cas des solutions plus précises et plus efficaces. Les capteurs confocaux chromatiques font parties des solutions astucieuses [1] permettant d'obtenir une résolution axiale submicronique sur tous les matériaux sans exception. Un intérêt supplémentaire est de proposer en complément de la mesure de distance, une mesure d'épaisseur sur les échantillons transparents [2].

\section{Principe d'imagerie confocale chromatique}

L'imagerie confocale chromatique s'appuie sur un ensemble d'optique présentant du chromatisme axial afin de coder l'information d'altitude et/ou d'épaisseur d'un objet positionné dans le champ de mesure. Ce système optique est également ancré dans une architecture confocale [3] afin d'accroître la sélectivité axiale et latérale. Le décodage de cette information se fait au travers d'un système de détection permettant de discriminer la ou les longueurs d'onde parfaitement focalisées sur la ou les surfaces de l'objet.

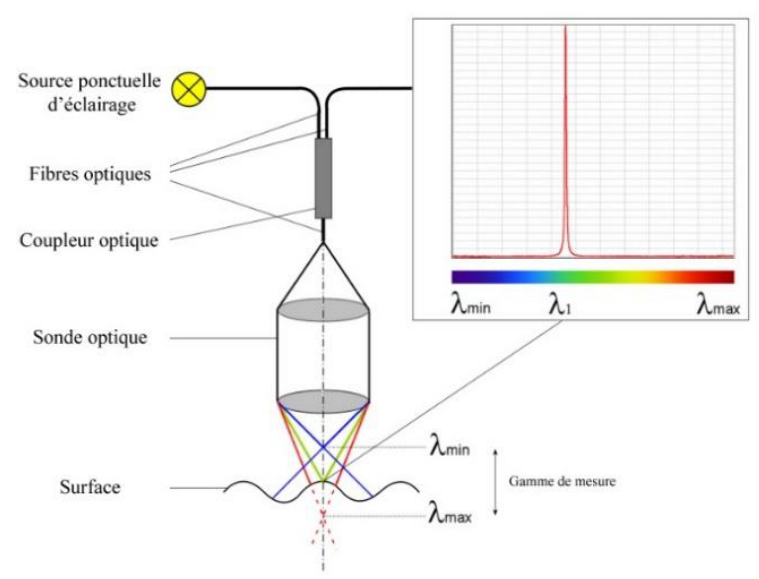

Figure 1 : Schéma de principe, mesure point.

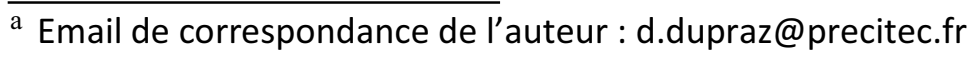


L'architecture standard d'un capteur confocal chromatique est composée de trois parties : un contrôleur électronique (source polychromatique, coupleur et spectromètre), une fibre optique de liaison et une sonde optique. Il existe deux configurations distinctes disponibles : mesure d'un point ou mesure d'un profil. Dans ce dernier cas, l'agencement de points consécutifs le long d'une ligne permet de mesurer, à travers une optique possédant du champ latéral, le profil d'un objet positionné dans le champ de mesure de la tête optique.

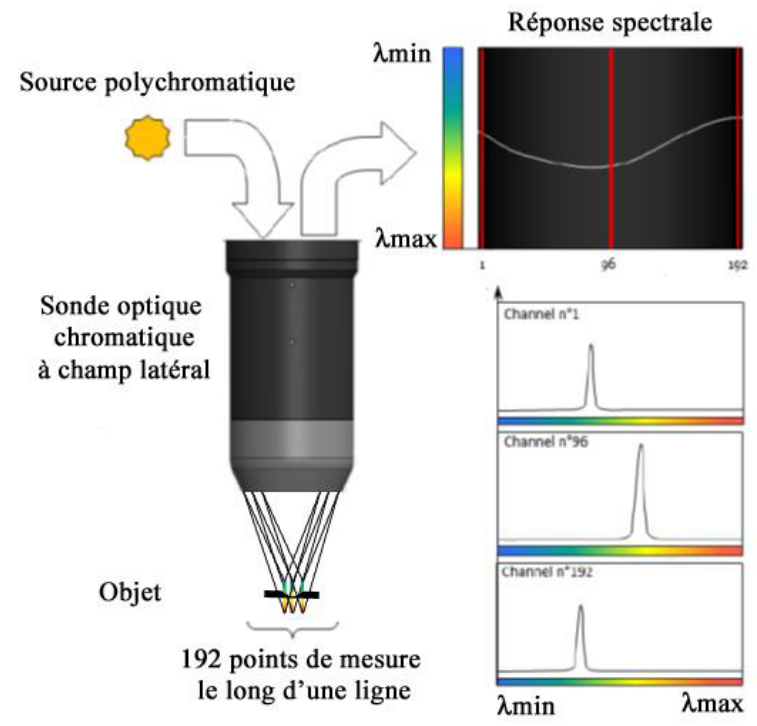

Figure 2 : Schéma de principe, mesure ligne.

Le schéma précédent présente une architecture avec 192 points de mesure simultanés repartis le long d'une ligne. Selon l'espacement entre chaque point, la longueur de ligne sera plus ou moins grande.

\section{CHRocodile CLS (Chromatic Line Sensor)}

\subsection{Concept tout-en-un}

Basée sur plus de 20 ans d'expérience dans le développement de capteurs et sondes optiques de type point, La société Precitec a développé un capteur de type ligne le CHRocodile CLS, composé de 192 points de mesure et utilisant le principe d'imagerie confocale chromatique. Son concept innovant est de proposer une architecture tout-en-un qui regroupe optique et électronique dans un même boîtier. Dans le cas d'un capteur dit ligne, cette architecture originale permet de s'affranchir d'un câble constitué d'une pluralité de fibres optiques (une ou deux fibres par voies de mesure). En complément et pour réduire au maximum l'encombrement, les coupleurs optiques sont remplacés par un séparateur de faisceau à l'intérieur du dispositif.

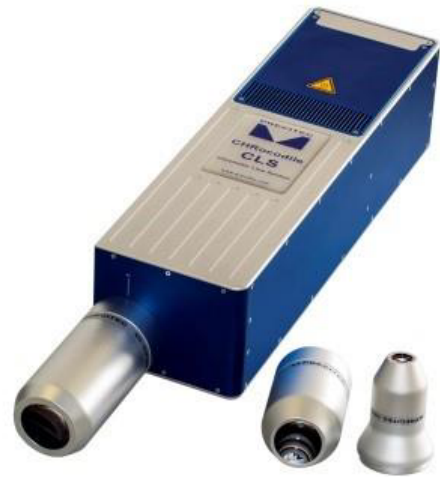

Figure 3 : Capteur ligne CHRocodile CLS avec sondes optiques interchangeables.

Cette innovation permet de surmonter les contraintes d'intégration du capteur en milieu industriel. Lorsque l'inspection doit être rapide et réalisée à $100 \%$ en production, la robustesse devient alors un critère primordial. L'absence de fibre optique de liaison lors de forte accélération et/ou de rotation est un atout majeur pour répondre efficacement aux problématiques du contrôle industriel. Afin d'adapter la gamme de mesure à l'application à résoudre, les objectifs chromatiques sont interchangeables offrant ainsi une modularité au système de mesure.

\subsection{Performances}

Le capteur CHRocodile CLS permet une mesure 3D incroyablement rapide de près de 400.000 points par seconde (profil de 192 points à une fréquence de $2 \mathrm{kHz}$ ) avec une résolution à l'échelle nanométrique. Une option permet d'augmenter la cadence de mesure jusqu'à 6000 profils/seconde en conservant la résolution axiale nominale.

\begin{tabular}{|c|l|l|l|}
\hline $\begin{array}{c}\text { Objectif } \\
\text { chromatique }\end{array}$ & \multicolumn{1}{|c|}{ CLS 0.2 } & CLS 1 & CLS 4 \\
\hline Gamme de mesure & $200 \mu \mathrm{m}$ & $0,95 \mathrm{~mm}$ & $3,9 \mathrm{~mm}$ \\
\hline Longueur de ligne & $0,96 \mathrm{~mm}$ & $1,91 \mathrm{~mm}$ & $4,78 \mathrm{~mm}$ \\
\hline Ecart entre points & $5 \mu \mathrm{m}$ & $10 \mu \mathrm{m}$ & $25 \mu \mathrm{m}$ \\
\hline Distance de travail & $5,3 \mathrm{~mm}$ & $18,5 \mathrm{~mm}$ & $39 \mathrm{~mm}$ \\
\hline Diamètre de spot & $2 \mu \mathrm{m}$ & $4 \mu \mathrm{m}$ & $10 \mu \mathrm{m}$ \\
\hline Résolution axiale & $20 \mathrm{~nm}$ & $80 \mathrm{~nm}$ & $320 \mathrm{~nm}$ \\
\hline $\begin{array}{c}\text { Angle max. sur un } \\
\text { miroir }\end{array}$ & $90^{\circ}+/-44^{\circ}$ & $90^{\circ}+/-33^{\circ}$ & $90^{\circ}+/-20^{\circ}$ \\
\hline
\end{tabular}

Table 1 : Performances en mode distance.

Très polyvalent, le CHRocodile CLS est également capable de mesurer l'épaisseur de matériaux transparents.

\begin{tabular}{|c|c|c|c|}
\hline $\begin{array}{c}\text { Objectif } \\
\text { chromatique }\end{array}$ & CLS 0.2 & CLS 1 & CLS 4 \\
\hline $\begin{array}{l}\text { Epaisseur } \\
\text { mesurable }\end{array}$ & $\begin{array}{l}20 \mu \mathrm{m}- \\
280 \mu \mathrm{m}\end{array}$ & $\begin{array}{c}75 \mu \mathrm{m}-1,35 \\
\mathrm{~mm}\end{array}$ & $\begin{array}{c}300 \mu \mathrm{m}-5,5 \\
\mathrm{~mm}\end{array}$ \\
\hline
\end{tabular}

Table 2 : Performances en mode épaisseur. 


\section{Applications}

Le capteur CHRocodile CLS ne connaît aucun compromis entre vitesse et précision - ce qui ouvre un tout nouveau domaine de mesures de topographie et de forme, que ce soit la hauteur, la largeur, la profondeur, l'angle, la rugosité ou la détection de défauts dans des industries telles que le semi-conducteur, l'aéronautique, la métallurgie ou la micromécanique, pour n'en citer que quelques-unes. Un exemple pratique a été réalisé avec un capteur CLS et son objectif chromatique CLS 1 (ligne de $1,91 \mathrm{~mm}$ ) monté sur une station 3 axes motorisés. L'objectif est de caractériser un dépôt d'encre sur une dalle de verre grâce à deux passes successives.

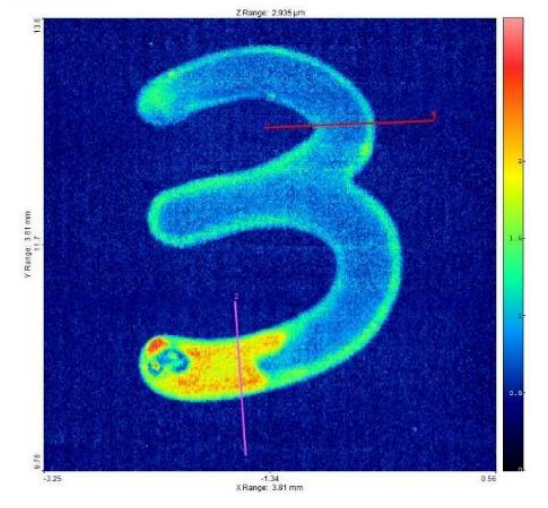

Figure 4 : Image d'altitude en fausse couleur, 3,81 x 3,81 mm avec un pas de $10 \times 10 \mu \mathrm{m}$. Temps d'acquisition : $0,8 \mathrm{~s}$.

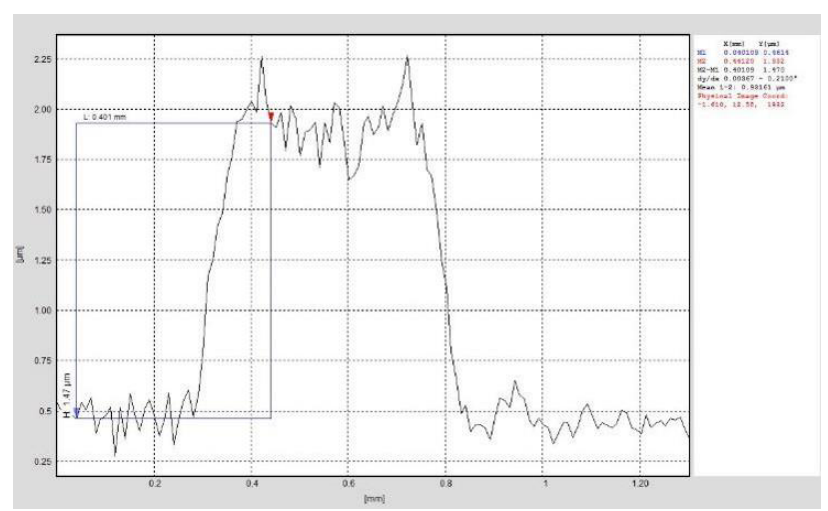

Figure 5 : Profil extrait de la figure précédente, hauteur d'encre mesurée de $1,47 \mu \mathrm{m}$.

Lors de l'analyse de la longueur d'onde parfaitement focalisée, il est possible d'extraire une information d'intensité directement reliée à la réflectivité de l'objet mesuré. A l'inverse d'une image provenant de la microscopie, la profondeur de champ est étendue car équivalente à la gamme de mesure de l'objectif chromatique utilisé (jusqu'à 3,9 mm). Ces données sont complémentaires à l'altitude et l'épaisseur pour identifier des défauts sur les surfaces à inspecter. Les systèmes 3D équipés d'un capteur point sont idéaux pour le laboratoire ou le bord de ligne de production lorsqu'il n'y a pas d'exigence sur des temps de cycle courts.

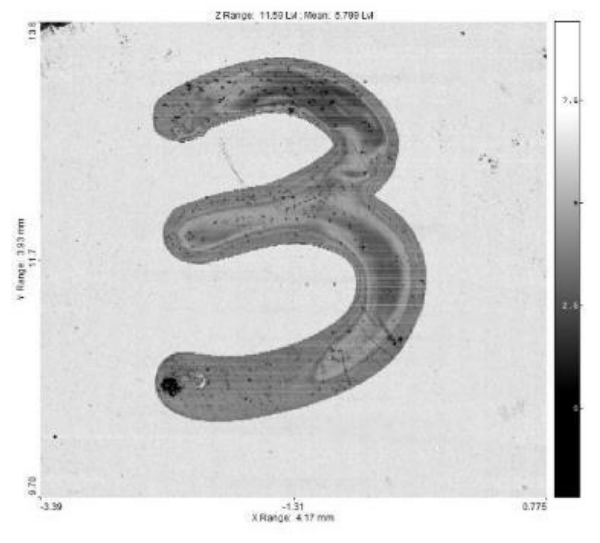

Figure 6 : Image d'intensité.

L'exemple traité a été mesuré de manière quasiinstantanée (inférieure à 1 seconde) avec le capteur ligne CHRocodile CLS. La mesure équivalente avec un capteur point aurait été de presque 3 minutes.

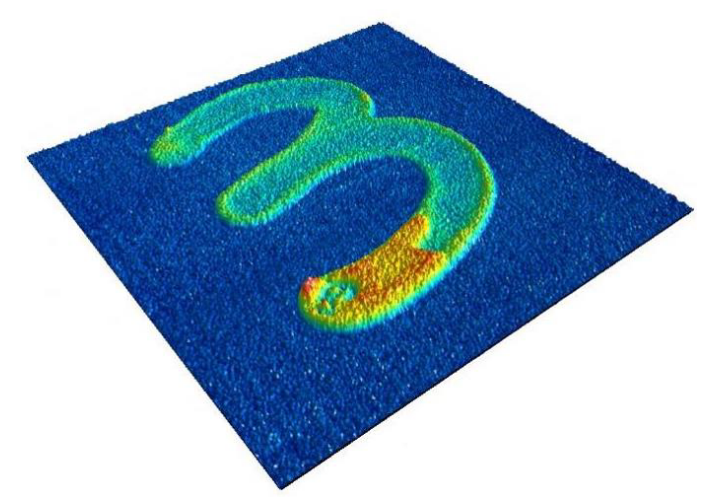

Figure 7 : Topographie 3D réalisée avec le logiciel SPIPC.

\begin{tabular}{|c|c|c|c|}
\hline $\begin{array}{c}\text { Objectif } \\
\text { chromatique }\end{array}$ & CLS 0.2 & CLS 1 & CLS 4 \\
\hline Applications & $\begin{array}{c}\text { Nano- } \\
\text { rugosité }\end{array}$ & $\begin{array}{c}\text { Planéité, état } \\
\text { de surface }\end{array}$ & Forme \\
\hline
\end{tabular}

Table 3 : Applications indicatives en fonction de l'objectif chromatique.

Le CHRocodile CLS est clairement le nouveau capteur de référence sur le marché et certainement le meilleur choix pour l'inspection ultra-rapide 3D de tous types de pièces. Ce capteur ouvre de nouvelles perspectives pour les industriels, dans des domaines requérants un instrument à la fois robuste, précis et rapide.

\section{Références}

1. G. Molesini, G. Pedrini, P. Poggi et F. Quercioli, Focus-wavelength encoded optical profilometer, Optics communications, 49 (1984)

2. M. Kunkel, J. Schulze, Glass science and technology, $8(2005)$

3. H.J. Tiziani, H.M. Udhe, Three-dimensional image sensing by chromatic confocal microscopy, Applied Optics, 33 (1994) 